\title{
Microvascular disease in chronic thromboembolic pulmonary hypertension: a role for pulmonary veins and systemic vasculature
}

\author{
Peter Dorfmüller ${ }^{1,2,3,8}$, Sven Günther ${ }^{1,3,4,8}$, Maria-Rosa Ghigna ${ }^{1,2,3}$, \\ Vincent Thomas de Montpréville ${ }^{2}$, David Boulate ${ }^{5}$, Jean-François Paul ${ }^{6}$, \\ Xavier Jaïs ${ }^{1,3,4}$, Benoit Decante ${ }^{5}$, Gérald Simonneau ${ }^{1,3,4}$, Philippe Dartevelle ${ }^{1,3,7}$, \\ Marc Humbert ${ }^{1,3,4}$, Elie Fadel ${ }^{1,3,5,7}$ and Olaf Mercier ${ }^{1,3,5,7}$
}

\begin{abstract}
Affiliations: ${ }^{1}$ Paris-South University, Faculty of Medicine, Kremlin-Bicêtre, France. ${ }^{2}$ Dept of Pathology, Centre Chirurgical Marie Lannelongue, Le Plessis-Robinson, France. ${ }^{3}$ INSERM U999, Pulmonary Hypertension: Pathophysiology and Novel Therapies, Centre Chirurgical Marie Lannelongue, Le Plessis-Robinson, France. ${ }^{4}$ National Reference Center of Pulmonary Hypertension, Dept of Pulmonology and Intensive Care Unit for Respiratory Diseases, Hôpital Bicêtre, AP-HP, Kremlin-Bicêtre, France. ${ }^{5}$ Surgical Research Lab, Centre Chirurgical Marie Lannelongue, Le Plessis-Robinson, France. ${ }^{6}$ Dept of Radiology, Centre Chirurgical Marie Lannelongue, Le Plessis-Robinson, France. ${ }^{7}$ Dept of Thoracic and Vascular Surgery and Heart-Lung Transplantation, Centre Chirurgical Marie Lannelongue, Le Plessis-Robinson, France. ${ }^{8}$ Both authors contributed equally to this study.
\end{abstract}

Correspondence: Peter Dorfmüller, Service d'Anatomie et de Cytologie Pathologiques, Centre Chirurgical Marie Lannelongue, 133 Avenue de la Résistance, 92350 Le Plessis-Robinson, France.

E-mail: peter.dorfmullerवu-psud.fr

ABSTRACT Limited numbers of operated patients with chronic thromboembolic pulmonary hypertension $(\mathrm{CTEPH})$ are refractory to pulmonary endarterectomy (PEA) and experience persistent pulmonary hypertension $(\mathrm{PH})$.

We retrospectively assessed lung histology available from nine patients with persistent $\mathrm{PH}$ (ineffective PEA (inPEA) group) and from eight patients transplanted for distal CTEPH inaccessible by PEA (noPEA group). Microscopically observed peculiarities were compared with the histology of a recently developed CTEPH model in piglets. Pre-interventional clinical/haemodynamic data and medical history of patients from the inPEA and noPEA groups were collected and analysed.

Conspicuous remodelling of small pulmonary arteries/arterioles, septal veins and pre-septal venules, including focal capillary haemangiomatosis, as well as pronounced hypertrophy and enlargement of bronchial systemic vessels, were the predominant pattern in histology from both groups. Most findings were reproduced in our porcine CTEPH model. Ink injection experiments unmasked abundant venular involvement in so-called small vessel or microvascular disease, as well as post-capillary bronchopulmonary shunting in human and experimental CTEPH.

Microvascular disease is partly due to post-capillary remodelling in human and experimental CTEPH and appears to be related to bronchial-to-pulmonary venous shunting. Further studies are needed to clinically assess the functional importance of this finding.

0 @ERSpublications

Pulmonary veins and systemic vasculature contribute to microvascular disease in CTEPH http://ow.ly/yGK4U

For editorial comments see page 1121.

This article has supplementary material available from erj.ersjournals.com

Received: Sept 272013 | Accepted after revision: June 132014 | First published online: Aug 192014

Conflict of interest: Disclosures can be found alongside the online version of this article at erj.ersjournals.com

Copyright @ERS 2014 


\section{Introduction}

Chronic thromboembolic pulmonary hypertension (CTEPH) is a severe disease that is life threatening if untreated [1]. CTEPH is represented in group four of the recent Nice Classification of Pulmonary Hypertension (former Dana Point Classification) [2,3]. The condition is probably underestimated, since clinical symptoms may vary and are not specific for CTEPH [4]. It has been reported that $1-3.8 \%$ of acute pulmonary embolism survivors develop CTEPH within 2 years of the first embolic event $[5,6]$.

Pulmonary endarterectomy (PEA) has replaced lung transplantation and is today the only curative treatment for patients with CTEPH $[7,8]$. However, only selected patients are eligible for PEA, depending on surgical accessibility of thromboembolic residues and underlying comorbidities [9]. It is well established that both obstruction of pulmonary arteries and secondary remodelling of small, peripheral pulmonary vessels (known as small vessel disease) most likely contribute to elevated total pulmonary resistance (TPR) $[10,11]$. Hence, discrepancies between vascular obstruction as assessed by radiology and haemodynamic severity are an important additional criterion for operability: patients with disproportionally high TPR unexplained by the vascular obstruction have a higher post-operative mortality [11]. It has been speculated that persistent pulmonary hypertension $(\mathrm{PH})$ after PEA, which represents the main cause of post-operative mortality, is partly due to remodelling of pulmonary microvessels inaccessible to surgical removal [12].

Our study addresses the role and importance of small vessel disease in CTEPH. To this purpose, we analysed histological, clinical, haemodynamic and functional features of CTEPH patients with persistent $\mathrm{PH}$ after PEA or heart-lung transplantation for inoperable CTEPH. In a proof-of-concept approach, observations from human CTEPH histology were compared with histology of a porcine CTEPH model that has been recently established in our institution $[13,14]$.

\section{Materials and methods \\ Patients}

We retrospectively reviewed clinical data and histological samples from 17 CTEPH patients that were transplanted between 1994 and 2010 either after PEA and persistent PH (ineffective PEA (inPEA) group) or because of primarily inoperable CTEPH (noPEA group). All CTEPH cases had been discussed preoperatively during interdisciplinary meetings in the presence of pulmonologists, thoracic surgeons and radiologists, and the final decision regarding PEA feasibility had been reached by consensus. All inPEA group patients had distal obstructive disease with high-level pulmonary resistance, but were considered to be operable. However, PEA resulted in persistent PH with right heart failure. All but one patient had cardiopulmonary assistance as a bridge to heart-lung transplantation in emergency. The noPEA group patients were initially deemed inoperable and eventually listed for transplantation. Clinical data are shown in table 1.

\section{Histology of human samples}

Lung samples from all patients were retrospectively analysed by conventional light microscopy. Lung tissue was systematically sampled after lung transplantation $(n=16)$ or during autopsy $(n=1)$ and eventually embedded in paraffin. 5- $\mu \mathrm{m}$ thick sections were stained with haematoxylin-eosin or haematoxylin-eosinsaffron, the latter staining highlighting collagen/fibrotic areas in orange colour. Chosen samples were submitted to elastin staining (Lannelongue quadrichrome staining: cytoplasm/smooth muscle in red, fibrin/ erythrocytes in magenta, collagen/fibrosis in blue, elastic fibres in black and nuclei in brown/black). Analysis corresponded to a semiquantitative score-based evaluation using a prospectively designed evaluation sheet with several histological items (tables 2 and 3). The resulting overall score of each item was not categorised, but displayed as an absolute value. Since assessment of bronchial artery hypertrophy was only possible on a few central lung samples, no sum was attributed to this item and results were expressed as the prevailing primary semiquantitative evaluation (scores 0-III). For details, please refer to the online supplementary material.

\section{Ink injection procedure of one human CTEPH case and of the porcine CTEPH model}

The lungs of one human CTEPH case, two CTEPH animals and one sham animal underwent an ex vivo ink injection procedure in order to trace the origin of remodelled microvessels and to reveal possible shunting between systemic and pulmonary vasculature. Briefly, green ink was injected into a main pulmonary vein at hilus level, blue ink was injected into the bronchial arteries, either at lung hilus level (human lung) or into the ostia at aortic level (porcine model). For details, please refer to the online supplementary material.

\section{Porcine CTEPH model}

We studied 10 Large White piglets weighing $21 \pm 3 \mathrm{~kg}$. The study complied with the Principles of Laboratory Animal Care developed by the National Society for Medical Research and was approved by our 
TABLE 1 Clinical data of chronic thromboembolic pulmonary hypertension patients

inPEA

\section{Subjects $n$}

Age years

Sex M/F n

Weight $\mathbf{k g}$

Height $\mathrm{cm}$

BMI $\mathrm{kg} \cdot \mathrm{m}^{-2}$

Pulmonary embolism

DVT

ICD

NYHA II

NYHA III

NYHA IV

6MWD m

FEV $1 \%$ predicted

TLC \% predicted

DLCO \% predicted

DLCO/V'A

CVP $\mathrm{mmHg}$

mPAP $\mathrm{mmHg}$

Cardiac index $\mathrm{L} \cdot \mathrm{min}^{-1} \cdot \mathrm{m}^{-2}$

TPR dyn $\cdot \mathbf{s} \cdot \mathrm{cm}^{-5}$

$\mathrm{SvO}_{2} \%$
noPEA

p-value

9
$47 \pm 6$
$4 / 5$
$63 \pm 2$
$170 \pm 3.5$
$22 \pm 0.8$
$5(55)$
$3(33)$
$4(44)$
0
$7(77)$
$2(23)$
$338 \pm 32$
$77 \pm 2.8$
$81 \pm 2.2$
$61.3 \pm 5$
$42.8 \pm 15$
$10 \pm 1.7$
$54.8 \pm 4$
$2.1 \pm 0.13$
$1251 \pm 157$
$53 \pm 3.7$

8
$47 \pm 2$
$5 / 3$
$67 \pm 2$
$173 \pm 2.4$
$22.46 \pm 0.6$
$3(37.5)$
$2(25)$
0
0
$1(12.5)$
$7(87.5)$
$331 \pm 51$
$81 \pm 3$
$90 \pm 8$
$69 \pm 2$
$59 \pm 2$
$12 \pm 2$
$51.2 \pm 1.9$
$2.2 \pm 0.08$
$1658 \pm 429$
$53.2 \pm 3.4$

NS

NS

NS

NS

NS

NS

NS

0.02

0.01

0.01

NS

NS

NS

NS

NS

NS

NS

NS

NS

NS

Data are presented as mean \pm SEM or $n(\%)$, unless otherwise stated. inPEA: ineffective pulmonary endarterectomy; noPEA: inoperable chronic thromboembolic pulmonary hypertension; M: male; F: female; BMI: body mass index; DVT: deep vein thrombosis; ICD: implanted cardiovascular device; NYHA: New York Heart Association; 6MWD: 6-min walking distance; FEV1: forced expiratory volume in $1 \mathrm{~s}$; TLC: total lung capacity; DLCO: diffusing capacity of the lung for carbon monoxide; V'A: alveolar ventilation; CVP: central vein pressure; mPAP: mean pulmonary artery pressure; TPR: total pulmonary resistance; $\mathrm{SvO}_{2}$ : mixed venous oxygen saturation; NS: nonsignificant.

local ethics committee on animal experiments. The piglets were randomly allocated to two groups of five animals each. Post-embolic PH was induced during 10 weeks in the experimental CTEPH group as previously described $[13,14]$. For details, please refer to the online supplementary material.

\section{Histology/morphometry of porcine samples}

After sacrifice, the lungs of all animals were collected, submitted to gross dissection and histology (haematoxylin-eosin-saffron staining), and eventually analysed by light microscopy. The left lung (ligation, complete occlusion), the right lower lobe (partial, progressive occlusion) and the right upper lobe (nonoccluded territory) were defined and differentiated as histological areas of interest. Results were compared to the left lung of sham-operated controls. Morphometric measurements were performed on one slide per area of interest from every CTEPH animal and on one slide of the left lung from all sham animals. For details, please refer to the online supplementary material.

\section{Statistical analysis}

Data are presented as mean \pm SEM unless stated otherwise. Demographic and medical characteristics were compared between groups using t-tests or Chi-squared tests depending on the variable type. Morphometric data of piglet histology were compared using ANOVA one-way and Tukey post-test analysis. A difference was considered significant when the p-value was $<0.05$. Statistical analysis was performed using Graphpad Prism 6 (Graphpad Software, San Diego, CA, USA).

\section{Results}

Demographic characteristics and medical history in CTEPH

All demographic characteristics of patients are shown in table 1. Both groups had similar characteristics except the pre-operative New York Heart Association (NYHA) class and the medical history of implanted cardiovascular device (ICD) use. Indeed, all patients were high-risk patients for surgery given the level of pre-operative pulmonary resistance and the reduced 6-min walking distance (6MWD). Notably, the noPEA 
TABLE 2 Histology score for the inPEA group ${ }^{\#}$

\begin{tabular}{|c|c|c|c|c|c|c|c|c|c|c|c|c|c|c|c|}
\hline Patient & AClF & AEIF & MH & RTL & OTL & PAI & PCHL & OED & IAS & VNF & SVF & PVI & $\mathbf{R I}$ & 이 & BAH \\
\hline TS & 0.3 & 1.2 & 0.1 & 0 & 0.7 & 0 & 0.1 & 0.2 & 0.3 & 0 & 0.1 & 0 & 0.5 & 1 & III \\
\hline FS & 0 & 0.7 & 0.1 & 0 & 0.6 & 0 & 1.4 & 0.7 & 0.7 & 0.9 & 1.3 & 0 & 0.2 & 0 & III \\
\hline MY & 0 & 0.3 & 1 & 0.4 & 0.7 & 0.6 & 0.5 & 0 & 0.6 & 2.2 & 0.6 & 1.8 & 0.4 & 0 & III \\
\hline LC & 0.6 & 1.4 & 0 & 0 & 0.5 & 0.3 & 0.8 & 0 & 0.6 & 1 & 0.4 & 0.5 & 0.3 & 0 & III \\
\hline DA & 0.4 & 2.3 & 0.3 & 0 & 1.4 & 0.4 & 0.2 & 0 & 0.2 & 2.2 & 2.1 & 0.9 & 0.2 & 0.6 & III \\
\hline Sum & 1.7 & 13 & 2.6 & 1.2 & 6.4 & 1.9 & 5.5 & 1.7 & 5.1 & 12.4 & 11.5 & 3.5 & 3.2 & 4.2 & \\
\hline
\end{tabular}

inPEA: ineffective pulmonary endarterectomy; ACIF: arterial concentric intimal fibrosis; AEIF: arterial eccentric intimal fibrosis; MH: medial hypertrophy; RTL: recent thrombotic lesion; OTL: organised thrombotic lesion; PAl: peri-arterial inflammation; PCHL: pulmonary capillary haemangiomatosis-like foci; OED: oedema; IAS: intra-alveolar siderophages; VNF: venular fibrosis; SVF: septal vein fibrosis; PVI: peri-venular inflammation; RI: recent infarctus; OI: organised infarctus; BAH: bronchial artery hypertrophy. The three items with the highest score (in bold) in this patient group were AEIF, a histological sign of peripheral organised thromboembolism, VNF and SVF. BAH was only assessed on central sections, when bronchial cartilage was present (three to eight slides per case for the inPEA group). Please refer to the extra material and methods section in the online supplementary material for details. ${ }^{\#}: n=9$.

group included more NYHA class IV patients, while the inPEA group presented more patients with a risk factor for ineffective surgical procedure (ICD use).

\section{Clinical, haemodynamic and lung functional characteristics in human CTEPH}

Results of NYHA functional class, 6MWD, pulmonary function tests and haemodynamic parameters are shown in table 1.

\section{Histological characteristics in human CTEPH}

The score-based histological evaluation of lung samples from the inPEA and noPEA groups is summarised in tables 2 and 3. The most prominent feature for both groups was the abundant presence of arterial eccentric intimal fibrosis concerning the majority of pre-capillary vessels. Organised thrombotic lesions, or so-called colander-like lesions, were frequently present. Intimal fibrosis of small pre-septal venules and larger septal veins was commonly found, with seven out of nine cases displaying both in the inPEA group, and all eight cases displaying both in the noPEA group. Interstitial remodelling with so-called pulmonary capillary haemangiomatosis-like foci was present in all nine inPEA and in seven out of eight noPEA cases.

TABLE 3 Histology score for the noPEA group ${ }^{\#}$

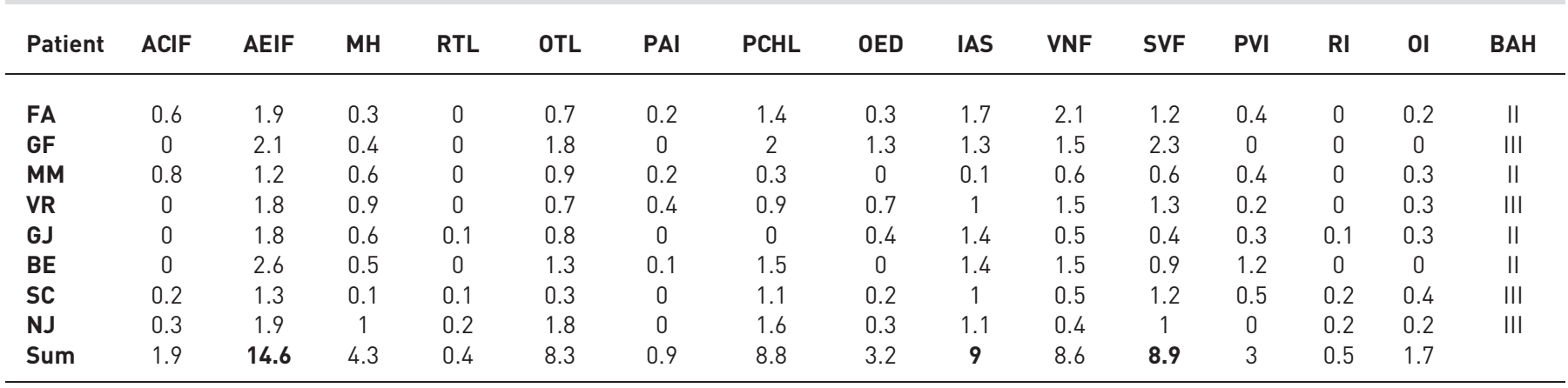

noPEA: inoperable chronic thromboembolic pulmonary hypertension; ACIF: arterial concentric intimal fibrosis; AEIF: arterial eccentric intimal fibrosis; MH: medial hypertrophy; RTL: recent thrombotic lesion; OTL: organised thrombotic lesion; PAl: peri-arterial inflammation; PCHL: pulmonary capillary haemangiomatosis-like foci; OED: oedema; IAS: intra-alveolar siderophages; VNF: venular fibrosis; SVF: septal vein fibrosis; $\mathrm{PVI}$ : peri-venular inflammation; RI: recent infarctus; OI: organised infarctus; BAH: bronchial artery hypertrophy. The three items with the highest score (in bold) in this patient group were AEIF, IAS and SVF. BAH was only assessed on central sections, when bronchial cartilage was present (three to five slides per case for the noPEA group). Please refer to the extra material and methods section in the online supplementary material for details. ${ }^{\#}: \mathrm{n}=8$. 
All cases presented muscular hypertrophy and enlargement of systemic bronchial arteries and vasa vasorum on samples from central lung areas. Typical histological findings are shown in figures 1-3.

Ink injection experiments in one patient from the inPEA group showed blue and green filling of peribronchial systemic vessels and microvessels (fig. 4a) and highlighted direct connections between small bronchial veins and pulmonary veins within lobular septa (fig. 4b). Mixed blue and green ink was observed in peripheral pulmonary arteries of the muscular type displaying eccentric intimal fibrosis (fig. 4c), as well as in recanalisation vessels within organised emboli of larger, elastic-type pulmonary arteries (fig. $4 \mathrm{~d}$ ). Green ink was found in remodelled pulmonary veins within lobular septa (fig. 4e) and in pre-septal venules (fig. 4f), as well as in remodelled microvessels, which proved to be post-capillary venules (fig. $4 \mathrm{~g}$ and $\mathrm{h}$ ). Alveolar capillaries adjacent to green ink-filled microvessels lacked significant ink marks, excluding the possibility of retrograde filling of pre-capillary arterioles.

\section{Haemodynamic characteristics in experimental porcine CTEPH}

Compared to sham animals, CTEPH animals had higher values for mean pulmonary artery pressure $(30.4 \pm 3.5$ versus $14 \pm 1 \mathrm{mmHg} ; \mathrm{p}=0.001)$ and TPR $\left(503 \pm 45\right.$ versus $\left.336 \pm 21 \mathrm{dyn} \cdot \mathrm{s}^{\cdot} \mathrm{cm}^{-5} ; \mathrm{p}=0.01\right)$. The haemodynamic results are summarised in table 4.

\section{Histological characteristics of experimental CTEPH}

Histological findings were similar in all five piglets of the CTEPH group. Compared with the sham group and in addition to the previously described post-obstructive vasculopathy, as well as the remodelling of muscular-type pulmonary arteries of unobstructed territories [13], animals of the CTEPH group displayed severe remodelling of septal veins within the left lung and, to a lower extent, within the right lower lobe (fig. 5a). Venous lesions included occlusive intimal fibrosis and arterialisation. Muscular remodelling of microvessels (arterioles or venules $<50 \mu \mathrm{m}$ in diameter) was present in decreasing proportions in CTEPH left lungs and CTEPH right lower lobes, while absent in CTEPH right upper lobes and sham animals (fig. 5b). Muscular hypertrophy and enlargement of bronchial arteries and vasa vasorum was impressive and present in similarly decreasing proportions within the left lungs and right lower lobes of all CTEPH animals, while right upper lobes and the lungs from sham animals did not display this pattern (fig. $5 \mathrm{c}$ ).

After injection of green ink into hilar veins and of blue ink into bronchial arteries in two CTEPH animals and one sham animal, we found that a significant proportion of remodelled/muscularised microvessels were of venous origin (venules). Also, large remodelled/arterialised septal vessels (anatomically veins) filled with green or with blue ink. Green pulmonary vein injection directly led to green filling of numerous bronchial veins, in the absence of significant alveolar capillary filling, and suggested a shunt between systemic vessels and septal pulmonary veins (fig. 6).

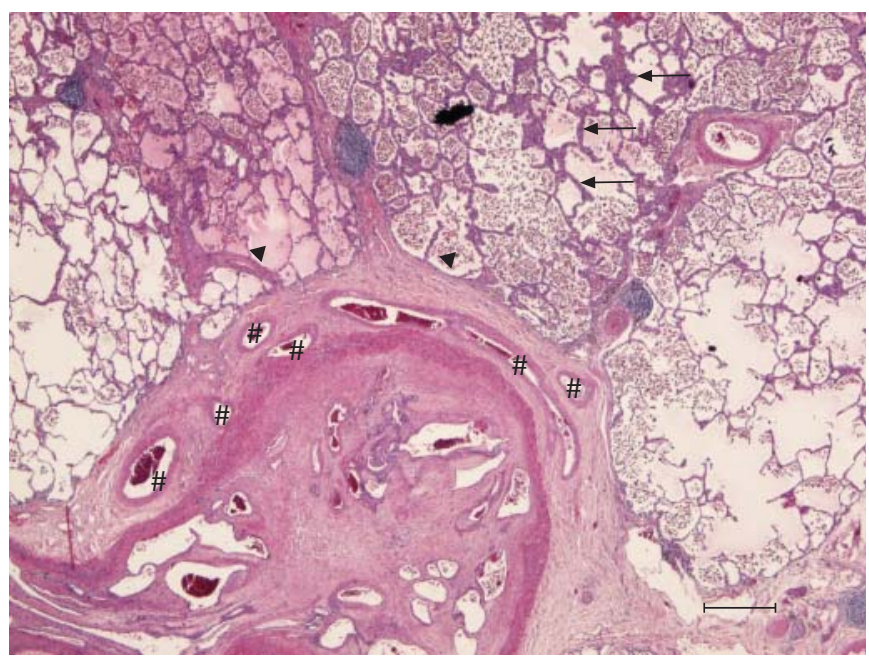

FIGURE 1 A lung sample of a patient suffering from chronic thromboembolic pulmonary hypertension (ineffective pulmonary endarterectomy group), stained with haematoxylin-eosin. A prominent, organised and partially repermeabilised thromboembolic lesion of a larger pulmonary artery is shown. The adjacent systemic vasa vasorum are numerous and show ectasia and hypertrophy (centre, ${ }^{\#}$ ). Also, note the thickening of the alveolar septa (top right, arrows), numerous intra-alveolar macrophages and focal oedema (top left, both indicated by arrowheads). Scale $\mathrm{bar}=500 \mu \mathrm{m}$. 

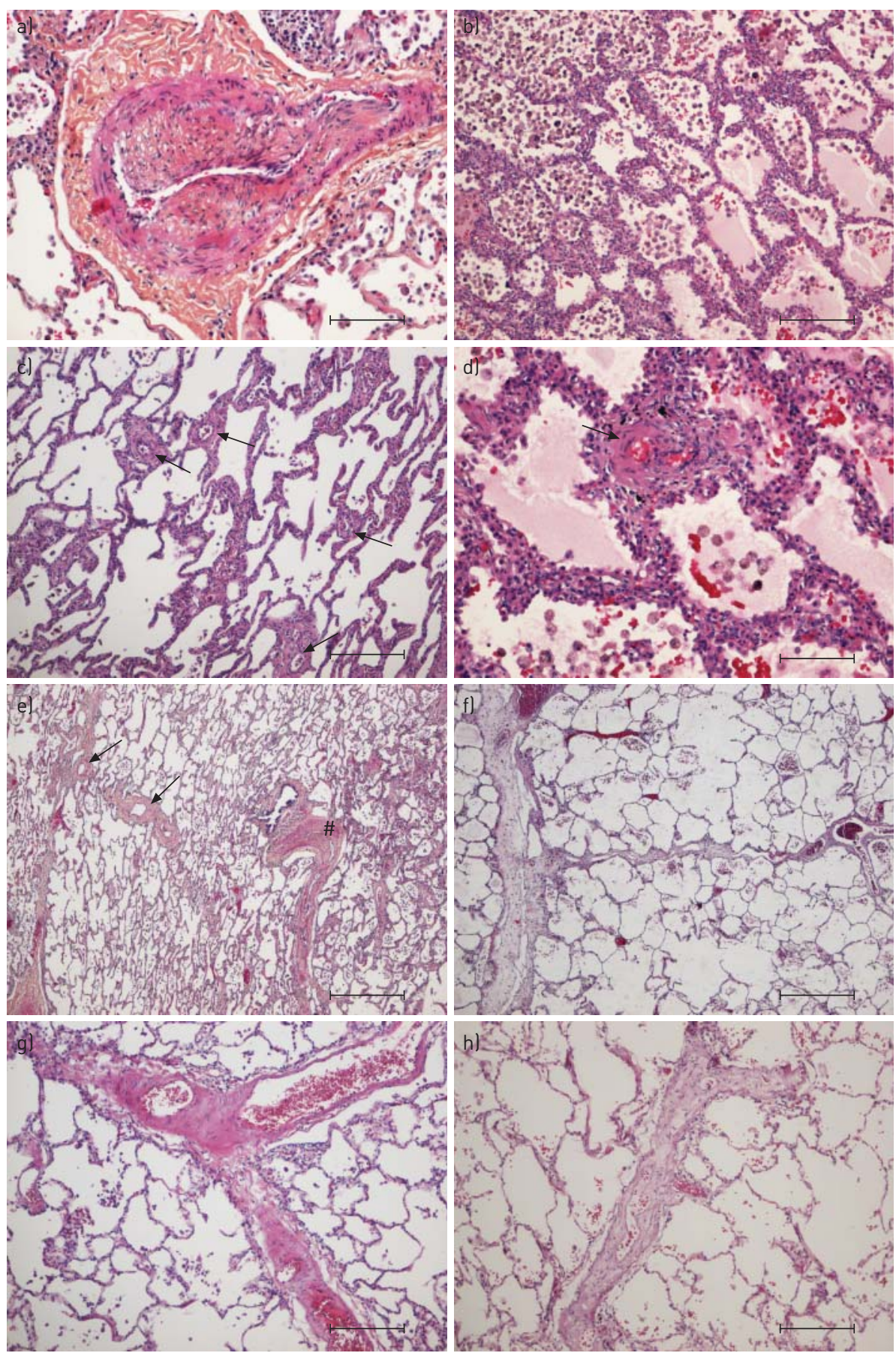

FIGURE 2 Lung samples of patients suffering from chronic thromboembolic pulmonary hypertension (CTEPH) (ineffective pulmonary endarterectomy group), stained with haematoxylin-eosin and haematoxylin-eosin-saffron. a) Small muscular pulmonary artery displaying eccentric intimal fibrosis, commonly seen in CTEPH. b) Alveolar septa are focally thickened by capillary congestion and multiplication, resembling the foci of pulmonary capillary haemangiomatosis. Note the high number of intra-alveolar macrophages/siderophages (left) and oedema (right). c) Small vessel disease: numerous pre- or post-capillary microvessels are visible and present muscular remodelling (arrows). d) Remodelled microvessel (arrow) and alveolar septal thickening. e) Overview comprising a muscular pulmonary artery with organised thrombotic lesions (right, ${ }^{\#}$ ) with its adjacent bronchiole, and remodelled veins displaying muscularisation and intimal fibrosis (left, arrows). f) Venules feeding into a septal vein, both displaying quasiocclusive loose intimal fibrosis. g) Partial occlusion of a septal vein by muscular remodelling and fibrosis. h) Venule with occlusive intimal fibrosis. Scale bars: a-c, g and h) $250 \mu \mathrm{m}$; d) $100 \mu \mathrm{m}$; e and f) $500 \mu \mathrm{m}$. 


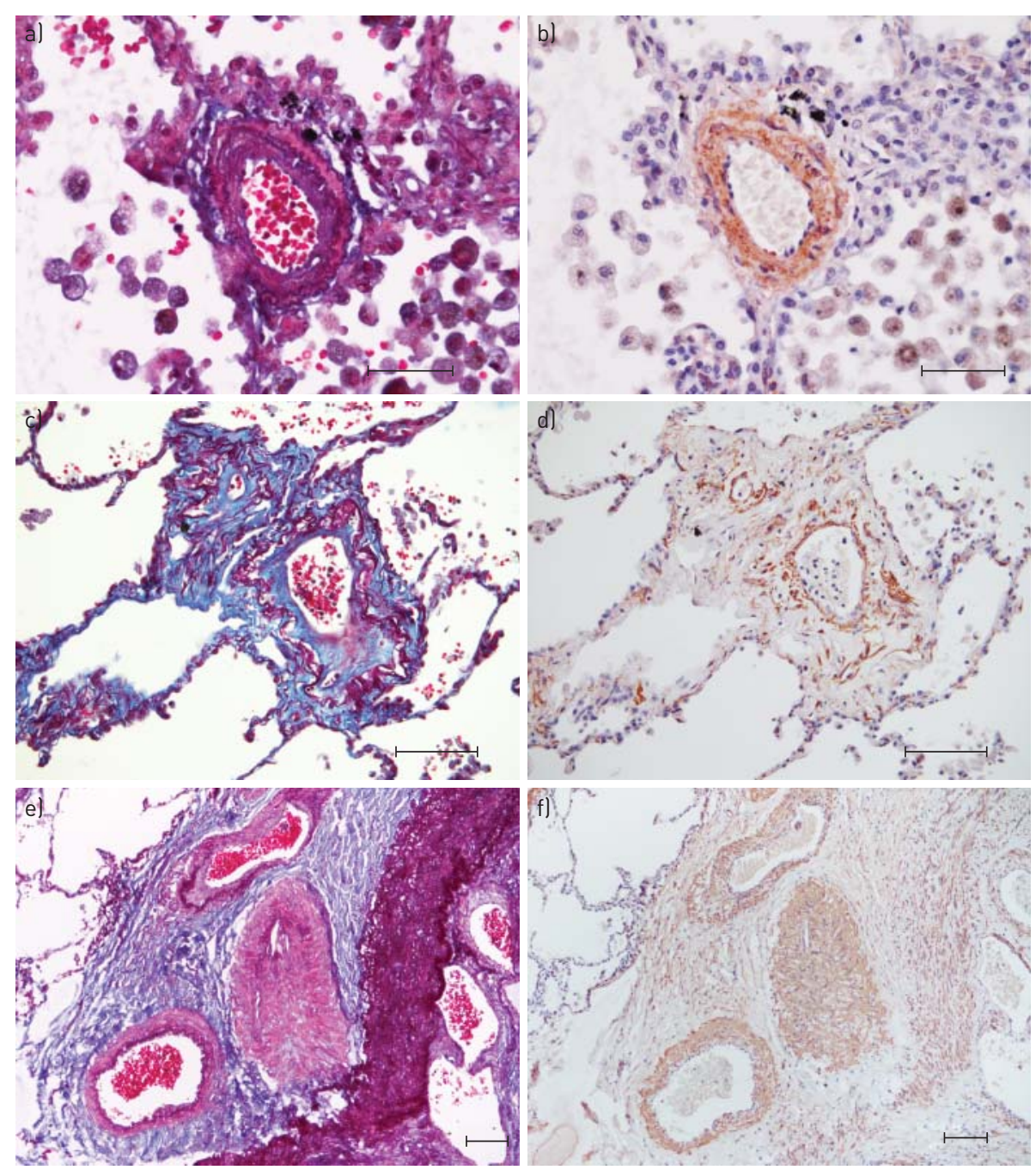

FIGURE 3 Lung samples of patients suffering from chronic thromboembolic pulmonary hypertension (ineffective pulmonary endarterectomy group). Lannelongue quadrichrome staining (cytoplasm/smooth muscle: red; fibrin/ erythrocytes: magenta; collagen/fibrosis: blue; elastic fibres: black; nuclei: brown) and smooth muscle cell immunostaining. $\mathrm{a}$ and b) Microvessel with well delimitated smooth muscle cell layer and intimal fibrosis (beyond the internal elastic lamina). c and d) Microvessel in vicinity of a septal vein (not shown) presenting with paucicellular, collagen-rich intimal fibrosis (blue) partly occluding the lumen; note the poorly developed muscularisation (actin staining). e and f) Vasa vasorum of a large pulmonary artery, which displays obliteration by organised thrombembolic material; the systemic vessels show smooth muscle cell hypertrophy/hyperplasia (actin staining) and a more or less well defined internal elastic lamina, while the external elastic lamina is either ill-defined or missing. Scale bars: a and b) $50 \mu \mathrm{m}$; c-f) $100 \mu \mathrm{m}$.

\section{Discussion}

Patients studied

The occurrence of pulmonary embolism and/or deep venous thrombosis (DVT) is a well-recognised criterion for the diagnosis of CTEPH and is a predictive factor for operable and proximal disease as well. However, most of the published series have reported a rate of $<50 \%$ for past medical history of pulmonary embolism or DVT in CTEPH patients [15-17]. As stated by PePKe-ZaBA et al. [18], the last report from the European registry has shown surprisingly high rates of pulmonary embolism and DVT history in these patients $(74.8 \%$ and $56.1 \%$, respectively), probably due to an increased awareness of thromboembolism in the participating centres. Our study reviewed charts from 17 CTEPH patients who experienced ineffective PEA or underwent transplantation for distal inoperable disease. Since all patients were bad candidates for surgery (e.g. PEA), we have probably selected patients with predominant microvascular disease and fewer proximal obstructive lesions. Consequently, the low incidence of pulmonary embolism history is consistent with our selection criteria for this study (table 1). 

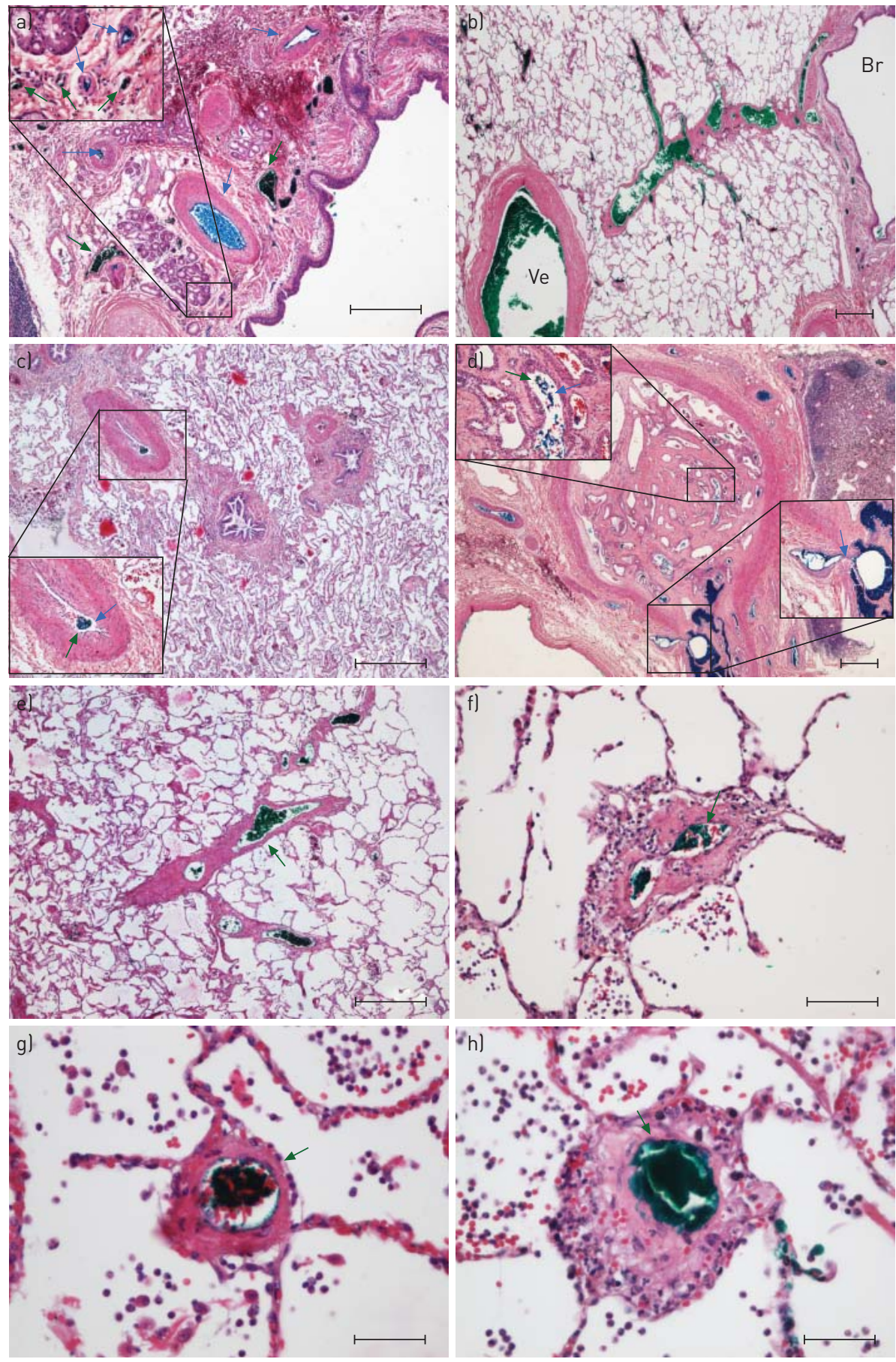

FIGURE 4 Lung samples from one chronic thromboembolic pulmonary hypertension (CTEPH) patient (ineffective pulmonary endarterectomy group) injected with blue (bronchial arteries) and green ink (pulmonary veins) during lung transplantation. a) Peri-bronchial vessels (arrows) are filled either with blue (artery-like, thick-walled vessels) or with green ink (vein-like thin-walled vessels); the magnified inset shows the same pattern on a microvascular level within the same bronchial wall. b) Green ink-filled vessels within a bronchial wall (right; Br) drain into a larger septal vein (centre), which in turn connects to a large (probably segmental), remodelled vein displaying muscularisation (left; Ve). c) Pulmonary arteries of the muscular type $(<500 \mu \mathrm{m})$ with eccentric intimal fibrosis showing intermixed blue and green ink within the narrowed lumen (magnified inset). d) Larger elastic-type pulmonary artery obstructed by organised thromboembolic material with numerous recanalisation vessels; the latter are filled with intermixed blue and green ink (magnified inset, top left). Note the visible connection to peri-arterial vasa vasorum highlighted with blue ink, suggesting a possible role for systemic vessels in resolving pulmonary emboli (magnified inset, bottom right). e) Remodelled septal veins displaying intimal fibrosis and muscularisation are filled with green ink. $\mathrm{f}-\mathrm{h}$ ) Several examples of remodelled microvessels. $\mathrm{f}$ and $\mathrm{h}$ ) Fibrotic thickening and some inflammatory infiltrate. $\mathrm{g}$ ) Muscularisation. All microvessels are filled out with green ink, identifying them as pulmonary venules and strongly suggesting involvement of venules in CTEPHassociated small vessel disease. Scale bars: a-e) $500 \mu \mathrm{m}$; f) $100 \mu \mathrm{m}$; g and h) $50 \mu \mathrm{m}$. 


\begin{tabular}{|c|c|c|c|}
\hline & Sham & CTEPH model & p-value \\
\hline Weight kg & $33.4 \pm 2$ & $41 \pm 5$ & 0.01 \\
\hline mPAP mmHg & $14 \pm 1$ & $30,4 \pm 3.5$ & 0.001 \\
\hline Cardiac output $\mathrm{L} \cdot \mathrm{min}^{-1}$ & $3.38 \pm 0.2$ & $4.98 \pm 0.6$ & 0.05 \\
\hline TPR dyn $\cdot \mathrm{s} \cdot \mathrm{cm}^{-5}$ & $336 \pm 21$ & $503 \pm 45$ & 0.01 \\
\hline CVP $\mathrm{mmHg}$ & $5 \pm \overline{0} .8$ & $8 \pm 1.5$ & NS \\
\hline $\mathrm{SvO}_{2} \%$ & $7 \overline{7} \pm 3$ & $70 \pm 4$ & NS \\
\hline Heart rate beats $\min ^{-1}$ & $88 \pm 5$ & $97 \pm 6$ & NS \\
\hline
\end{tabular}

Data are presented as mean \pm SEM unless otherwise stated. MPAP: mean pulmonary artery pressure; TPR: total pulmonary resistance; CVP: central venous pressure; $\mathrm{SvO}_{2}$ : mixed venous oxygen saturation; NS: nonsignificant.

Both groups represented high-risk patients for surgery, with pulmonary resistance $>900 \mathrm{dyn} \cdot \mathrm{s} \cdot \mathrm{cm}^{-5}$, $6 \mathrm{MWD}<350 \mathrm{~m}$, NYHA III or IV, lack of thromboembolic disease past history and/or use of ICDs. Both groups were at high risk for surgery but the noPEA group was at higher risk given the NYHA status.

\section{Findings in human histology}

We report involvement of distal muscular-type pulmonary arteries and microvessels, pronounced venous remodelling and haemangiomatosis-like foci in lung samples from CTEPH patients. Our findings suggest a pathogenic contribution of the peripheral pre-capillary and, more surprisingly, of the post-capillary vasculature. We have compared our findings with a recently established porcine model of CTEPH and report significant venular involvement in so-called small vessel disease of CTEPH, associated with increased bronchopulmonary shunting.

\section{Pulmonary arteries}

Classic thromboembolic remodelling of larger arteries of the elastic type has been reported to be associated with secondary vascular lesions of distal, surgically inaccessible small pulmonary vessels [12]. A chronic, if not progressive character has been attributed to this so-called small vessel disease, probably accounting for the observed increase of PH in CTEPH patients lacking any detectable secondary embolic events [12, 19, 20]. Moreover, remodelling of small peripheral vessels beyond surgical accessibility may be a major contributor to persistent PH after PEA [7]. Indeed, we found that all lungs of analysed CTEPH patients displayed peripheral arterial remodelling. Eccentric fibrous thickening of the intima was the most frequently observed histological item and was present on all slides from peripheral areas (tables 2 and 3). In their descriptive analysis of $31 \mathrm{CTEPH}$ patients, MOSER and BLOOR [10] have likewise reported eccentric intimal thickening as the most prominent histological feature in peripheral muscular arteries, followed by concentric intimal

a)

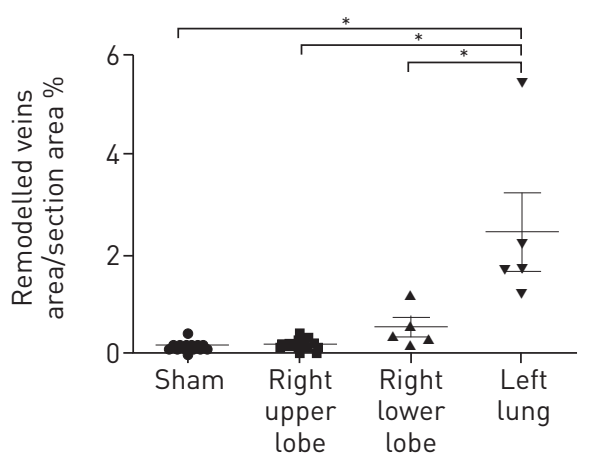

b)

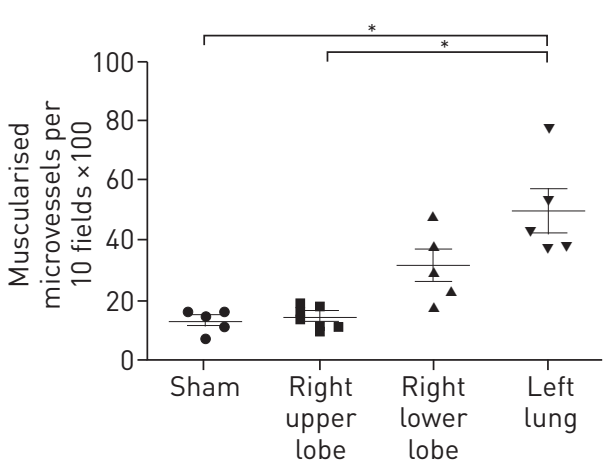

c)

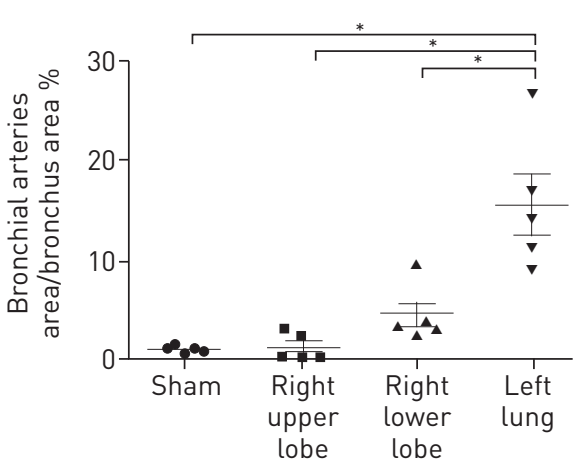

FIGURE 5 Degree of a) pulmonary venous, b) pulmonary microvessel $(<50 \mu \mathrm{m})$ and c) bronchial arterial remodelling in lungs from sham animals and in the right upper lobe (unobstructed area), right lower lobe (repeatedly embolised area) and left lung (ligation, totally occluded area) of chronic thromboembolic pulmonary hypertension animals. Please refer to the extra material and methods section in the online supplementary material. Note the concordance of these three histological patterns in all four groups, suggesting a pathophysiological dependence. ${ }^{*}: \mathrm{p}<0.05$. 


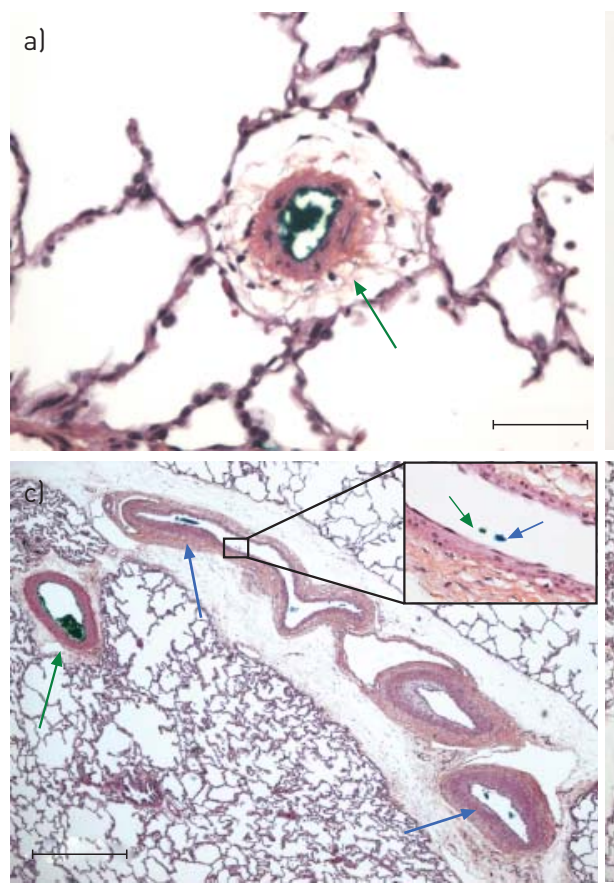

b)
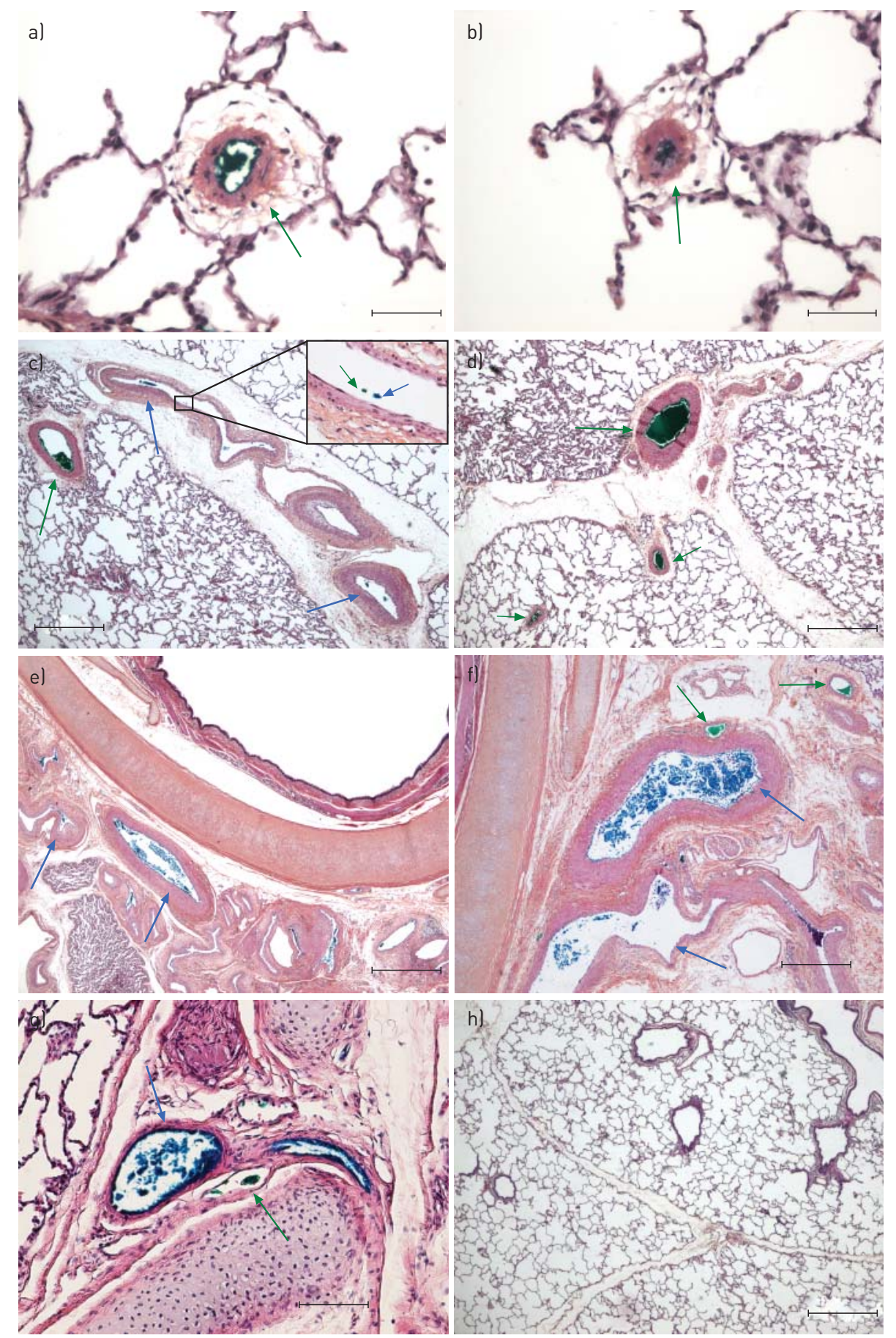

FIGURE 6 Chronic thromboembolic pulmonary hypertension model in piglets. Remodelling of pulmonary microvessels, vessels within lobular septa and bronchial vessels. Haematoxylin-eosin-saffron staining with blue (bronchial arteries) and green (pulmonary veins) ink injection. a and b) Strongly remodelled microvessels displaying muscularisation and adventitial broadening partly turn out to be post-capillary venules: the lumen is filled out by green ink (green arrows), which has been injected retrogradely into the proximal (hilar) pulmonary veins. c) Intense muscular and fibrotic remodelling in vessels within a lobular septum. The pre-septal vein (strong muscular thickening) shows green ink filling (green arrow), intra-septal remodelled vessels show blue and green ink traces (blue and green arrows), and the magnified inset shows intermingled blue and green ink. d) Green ink filling within remodelled intra- and pre-septal veins (green arrows). e) Multiplication and muscular hypertrophy of systemic bronchial arteries (blue ink filling, blue arrows). f) The bronchus wall shows blue ink-filled arteries (blue arrows) and green ink-filled veins (green arrows), probably bronchial veins that are connected to the injected pulmonary veins. g) Sham animal: small, normal-sized bronchial arteries fill with blue ink (blue arrow), thin-walled bronchial veins display green ink (green arrow), and the connection appears to be present under physiological conditions. h) Overview of a lung sample from a sham piglet. Note the slender lobular septa, lacking venous remodelling. Scale bars: a and b) $50 \mu \mathrm{m}$; c, d, f and h) $500 \mu \mathrm{m}$; e) $1000 \mu \mathrm{m}$; g) $100 \mu \mathrm{m}$. 
thickening. In contrast to the study from MOSER and BLOOR [10], we did not find significant evidence for plexiform lesions in our series. In the past, plexiform lesions have not always been perceived as a feature of CTEPH [21]. However, other groups have reported such lesions in CTEPH, as well as in several forms of pulmonary arterial hypertension $(\mathrm{PAH})$, and the discussion remains controversial $[10,22,23]$.

\section{Pulmonary veins and capillaries}

Our histological findings suggest involvement of post-capillary pulmonary vessels. When present, fibrous lesions were observed on most slides, but intensity was moderate and did not reach the importance/ predominance of venous remodelling in pulmonary veno-occlusive disease (PVOD). Most importantly, ink injection experiments highlighted numerous small typically remodelled microvessels in green, unmasking them as venules and suggesting pulmonary venular involvement in CTEPH-associated small vessel disease (fig. 4). Our elastin stainings and immunohistochemical experiments highlighting microvascular smooth muscle cells confirmed this deduction (fig. 3). It should be outlined that the combination of gentle ink injection and elevated viscosity of the dye as compared to blood prevented the capillary bed from filling in a retrograde fashion. Significant backfilling of arterioles through the capillary bed with green ink could thus be excluded. The described handling and dye properties were probably also responsible for significant ink concentrations in vessels before a shunt and only very small quantities beyond the shunting point, as seen for green ink in pulmonary arteries and blue ink in pulmonary veins/arteries. Capillary haemangiomatosislike areas were present, although not as regularly encountered as is the case in PVOD. Notably, distribution of these foci was different compared with PVOD: we observed close topographical association with segmental or subsegmental pulmonary arteries displaying organised arterial thromboembolic obstruction. In contrast, PVOD is characterised by an intra-parenchymal patchy distribution of haemangiomatosis-like foci, irrespective of larger arteries [24]. To our knowledge, this venous and capillary pattern is described for the first time in human CTEPH.

Intrapulmonary systemic vessels

All lungs from the inPEA and noPEA groups had pronounced hypertrophy of bronchial vessels and vasa vasorum. Peri-bronchial arteries displayed muscular thickening and both systemic arteries and veins were enlarged. In our ink-injected case, we found bronchial systemic vessels highlighted either in blue or in green. Our experiments confirm what is known: bronchial vessels are directly connected to post-capillary pulmonary vessels [25]. However, our ink experiments illustrate that large (green) bronchial/pulmonary veins are localised within the adventitia of the bronchus, directly adjacent to the (blue) bronchial arteries. We did not perceive a well-developed "bronchial capillary bed", which might suggest that the connections resemble more or less direct connections/anastomoses between bronchial arterioles and pulmonary veins, which are only significantly increased and highly functional when necessary, e.g. in a condition with bronchial arterial hypertrophy. Recanalisation vessels within organised pulmonary arterial emboli, as well as peripheral pulmonary arteries displaying eccentric intimal fibrosis, showed intermixture of green and blue ink, suggesting not only a direct connection between systemic and pulmonary arteries, but also between the latter and pulmonary veins, in other words pulmonary arteriovenous shunting. Although we will not discuss this point extensively, since these findings were sparse in our analysis, the phenomenon has been shown for humans and animals under exercise conditions [26-28]. The pattern of hypertrophy and enlargement of systemic bronchial arteries is well known in CTEPH $[25,29]$. It has been speculated that a decrease of postobstructive pulmonary artery pressure and the eventual increase of the pressure gradient between bronchial arteries and pulmonary arteries might lead to an opening of pre-existing bronchopulmonary arterial anastomoses [30, 31]. Interestingly, JOHNSON et al. [32] reported a significant increase in number of muscularised veins as well as an increase of wall thickness of pulmonary veins in sheep with $\mathrm{PH}$ induced by continuous air embolisation into the main pulmonary artery. The authors also found an increase in volume density of small bronchial vessels compared with controls, at day 12 of continuous air embolisation [32]. Since anastomoses between bronchial artery branches and pre-capillary pulmonary arterioles and also between bronchial arteries and pulmonary veins have been described in the past, a direct systemic impact on post-capillary vessels and on the capillary network itself appears possible $[25,33,34]$. In this respect, the unprecedented quantitatively significant connection of elevated pressures in the systemic circuit with a pulmonary venous low-pressure system would explain an extensive reaction of venous wall compartments, comprising muscularisation of the media and fibrotic thickening of the intima, as well as a consecutive congestive repercussion on the capillary network, eventually leading to capillary proliferation. In addition, remodelling within the capillary bed but also muscular thickening of pre-capillary arterioles could partly be due to an opening of the previously described bronchopulmonary arterial anastomoses. The focal distribution of this mechanism, which is triggered by arterial embolic occlusion and hence occurs in the vicinity of occluded pulmonary arteries, would account for the pathological vessel wall reaction in response 
to a focal, disproportionally strong pressure stimulus. This proposed mechanism is schematically outlined in figure 7.

\section{Findings in porcine histology}

Within the left lung and the right lower lobe, all CTEPH animals $(n=5)$ displayed important muscular hypertrophy, enlargement and increase in number of bronchial arteries and vasa vasorum. Muscularisation of microvessels was constantly present, while only sporadically present in the right upper lobe and in sham controls. Inter-group comparison of sham animal lungs, right upper lobe of CTEPH animals (no occlusion), right lower lobe (partial and progressive occlusion) and left lung (ligation, complete occlusion from day 1) revealed significant differences regarding the degree of pulmonary venous remodelling, bronchial artery hypertrophy and microvascular muscularisation, suggesting a functional concordance of these three vascular changes. The peak of changes in these three morphological parameters appeared to be in the area of total pulmonary arterial occlusion through ligation, while partial and progressive occlusion through repeated emboli generated the same modifications, but to a lesser extent. This observation, at least for bronchial arterial remodelling, is in accordance with findings from a study by SHIMIZU et al. [29], whose retrospective analysis of computed tomography angiography from 59 patients with CTEPH showed that the cross-sectional area of the bronchial arteries positively correlated with the central extent of the thromboembolic material compared with obstruction on the segmental level. They also compared the

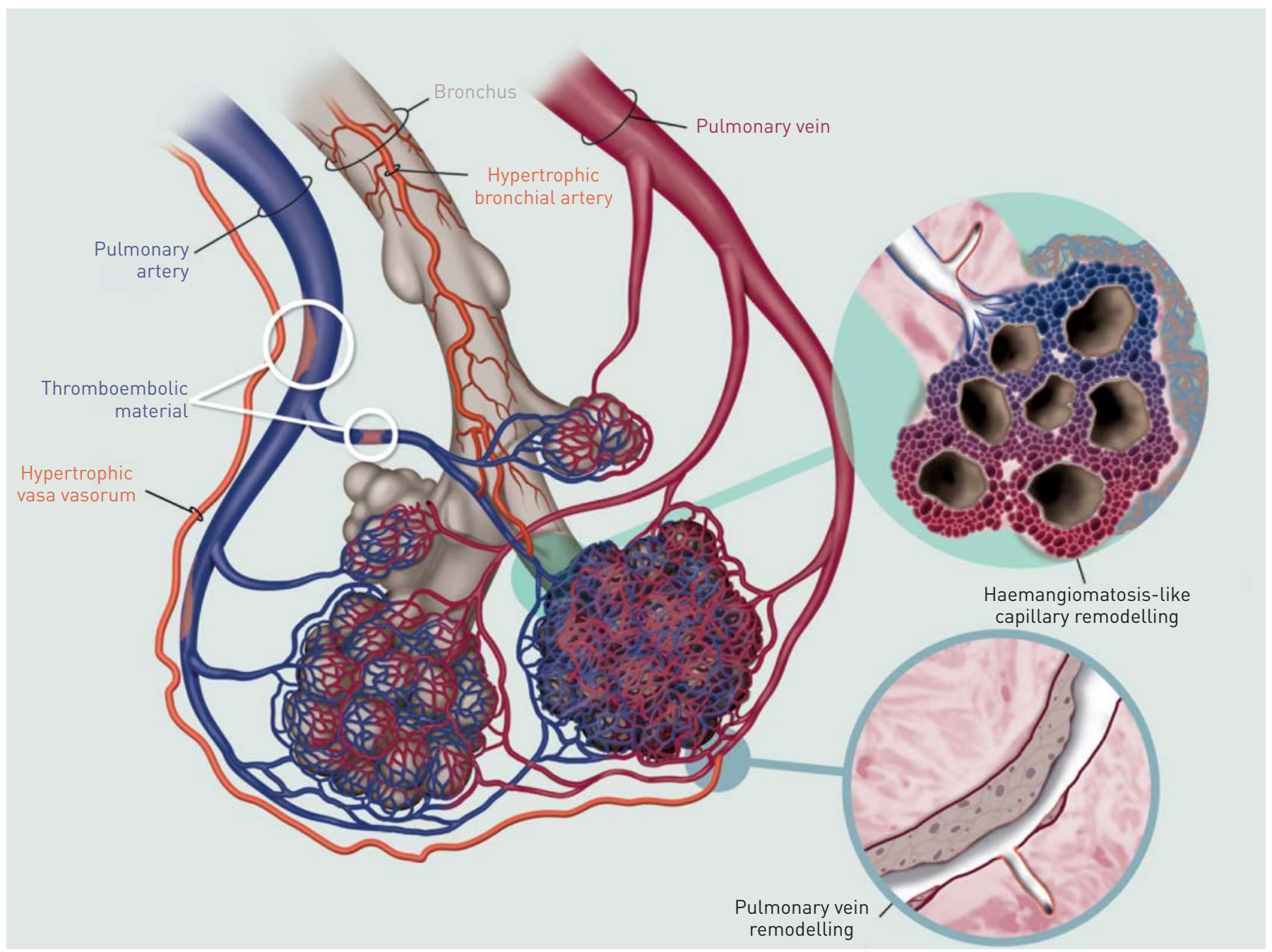

FIGURE 7 A proposed mechanism for bronchopulmonary venous (and arterial) shunting, as demonstrated in human and experimental chronic thromboembolic pulmonary hypertension. Note the schematic pre-capillary connections of systemic arteries (represented as bronchial arteries and vasa vasorum) with pulmonary arteries and with post-capillary pulmonary veins/venules. One magnified inset (top) shows the reactive thickening of the pulmonary arteriole and a haemangiomatosis-like focus featuring a multiplication of alveolar capillaries just beyond the bronchopulmonary arterial shunt. The other magnified inset (bottom) shows reactive fibrotic remodelling of a pulmonary vein wall at the level of a bronchopulmonary venous connection, relating systemic pressure with the low-pressure venous system of the lung. 
CTEPH group to 16 patients suffering from PAH (group 1 of the Nice classification) and found significant increase in bronchial artery total area of CTEPH patients compared with PAH. However, no significant difference of bronchial artery total area between distal (segmental) type CTEPH and PAH was detected. Interestingly, 24 patients of the CTEPH group underwent PEA and 21 were eventually submitted to postPEA computed tomography angiography, which showed significant decrease in bronchial artery total area. These results reflect our experimental finding of a positive correlation between the central extent of thromboembolic obstruction and the hypertrophy of bronchial arteries. Moreover, they illustrate the reversibility of bronchial artery hypertrophy in CTEPH.

Additionally, it has been shown at an experimental level that ligation of the left pulmonary artery in newborn lambs leads, after 1-3 years, to hypertrophy of the systemic bronchial vasculature along the bronchial branches, forming a dense vascular plexus and eventually filling the pulmonary capillaries [35]. The anatomical analysis was performed through macroscopic observation of a bronchial arterial tree cast. Our observations in young piglets corroborate with these results regarding hypertrophy of bronchial vessels, even as early as 10 weeks after left pulmonary artery ligation. Importantly, our pulmonary venous retrograde injected green ink did not pass through the alveolar capillary network but directly into bronchial veins, suggesting an unfiltered connection of bronchial blood flow and pulmonary veins. This observation is endorsed by a recent echographic study on anaesthetised sheep using gelatin microballoons for echocontrast [36]. From their observations, PARSONs et al. [36] infer that there are bronchopulmonary anastomoses beyond the alveolar capillaries that connect the bronchial circulation to the pulmonary venous circulation. These observations are notable, since CHARAN et al. [37] have experimentally shown that by far the major portion of bronchial arterial blood drains into the pulmonary circulation and only about $13 \%$ returns systemically to the right heart. Hence, a significant flow impact on pulmonary veins due to systemic to pulmonary post-capillary anastomoses appears conceivable. Indeed, this mechanism would explain the close correlation of all three morphological parameters in our porcine model: increase of venous and microvascular remodelling due to increase of bronchial arterial flow/hypertrophy.

Moreover, in our porcine model we found bronchial artery-specific blue ink filling in large muscularised vessels running within lobular septa in direct vicinity to green ink-filled pulmonary septal veins, as well as beneath the visceral pleura. The latter observation corroborates with results from the study of CHARAN and CARVALHO [35] in lambs: in their left pulmonary artery ligation model, they describe enlargement of vessels that supply the visceral pleura, which are partially directly connected to bronchial vessels supplying the terminal airways. This finding is of particular interest, since in normal human lungs bronchial arteries are not known to run within lobular septa; the latter contain pulmonary veins and lymphatic vessels [34, 38]. Hence, in this setting, an arterialisation of septal veins, either pulmonary or bronchial, can be assumed. Although signs of congestion and mild thickening of alveolar septa in some porcine lung areas were present, we did not find a full-grown pattern of capillary haemangiomatosis-like areas in our CTEPH model. The reason for this difference from human CTEPH probably lies in the lack of real chronicity of our model, since all piglets were sacrificed after 10 weeks.

\section{Conclusion}

Our study on human CTEPH, comprising a histological comparison with a recently developed porcine model, confirms the importance of small vessel or microvascular disease in lungs from inoperable patients or with persistent PH after PEA. We have newly identified a prominent involvement of the post-capillary pulmonary venous compartment in CTEPH-associated small vessel disease and were able to reproduce these modifications in experimental porcine CTEPH. Post mortem ink injection procedures in one human CTEPH case and in our model allowed us to literally connect post-capillary venous remodelling to the hypertrophic systemic bronchial vasculature. We conclude that bronchopulmonary venous shunting is present and may be of importance in the pathophysiology of human CTEPH. Prospective studies are needed in order to further substantiate this suspicion and eventually to develop imaging tools that may quantify the involvement of microvascular disease in vivo.

\section{References}

1 Pepke-Zaba J. Diagnostic testing to guide the management of chronic thromboembolic pulmonary hypertension: state of the art. Eur Respir Rev 2010; 19: 55-58.

2 Simonneau G, Robbins IM, Beghetti M, et al. Updated clinical classification of pulmonary hypertension. J Am Coll Cardiol 2009; 54: Suppl. 1, S43-S54.

3 Simonneau G, Gatzoulis MA, Adatia I, et al. Updated clinical classification of pulmonary hypertension. J Am Coll Cardiol 2013; 62: Suppl. 25, D34-D41.

4 Madani MM, Auger WR, Pretorius V, et al. Pulmonary endarterectomy: recent changes in a single institution's experience of more than 2,700 patients. Ann Thorac Surg 2012; 94: 97-103.

5 Pengo $\mathrm{V}$, Lensing $\mathrm{AW}$, Prins $\mathrm{MH}$, et al. Incidence of chronic thromboembolic pulmonary hypertension after pulmonary embolism. N Engl J Med 2004; 350: 2257-2264. 
6 Becattini C, Agnelli G, Pesavento R, et al. Incidence of chronic thromboembolic pulmonary hypertension after a first episode of pulmonary embolism. Chest 2006; 130: 172-175.

7 Jamieson SW, Kapelanski DP, Sakakibara N, et al. Pulmonary endarterectomy: experience and lessons learned in 1,500 cases. Ann Thorac Surg 2003; 76: 1457-1462.

8 Lausberg HF, Tscholl D, Schäfers HJ. Pulmonale Thrombendarteriektomie [Pulmonary thromboendarterectomy]. Anaesthesist 2004; 53: 748-752.

9 Jenkins DP, Madani M, Mayer E, et al. Surgical treatment of chronic thromboembolic pulmonary hypertension. Eur Respir J 2013; 41: 735-742.

10 Moser KM, Bloor CM. Pulmonary vascular lesions occurring in patients with chronic major vessel thromboembolic pulmonary hypertension. Chest 1993; 103: 685-692.

11 Hoeper MM, Mayer E, Simonneau G, et al. Chronic thromboembolic pulmonary hypertension. Circulation 2006; 113: 2011-2020.

12 Galiè N, Kim NH. Pulmonary microvascular disease in chronic thromboembolic pulmonary hypertension. Proc Am Thorac Soc 2006; 3: 571-576.

13 Mercier O, Fadel E. Chronic thromboembolic pulmonary hypertension: animal models. Eur Respir J 2013; 41 : $1200-1206$.

14 Mercier O, Tivane A, Dorfmüller P, et al. Piglet model of chronic pulmonary hypertension. Pulm Circ 2013; 3: 908-915.

15 Wolf M, Boyer-Neumann C, Parent F, et al. Thrombotic risk factors in pulmonary hypertension. Eur Respir J 2000; 15: 395-399.

16 Bonderman D, Jakowitsch J, Adlbrecht C, et al. Medical conditions increasing the risk of chronic thromboembolic pulmonary hypertension. Thromb Haemost 2005; 93: 512-516.

17 Lang IM. Chronic thromboembolic pulmonary hypertension - not so rare after all. N Engl J Med 2004; 350: 2236-2238.

18 Pepke-Zaba J, Delcroix M, Lang I, et al. Chronic thromboembolic pulmonary hypertension (CTEPH): results from an international prospective registry. Circulation 2011; 124: 1973-1981.

19 Moser KM, Auger WR, Fedullo PF, et al. Chronic thromboembolic pulmonary hypertension: clinical picture and surgical treatment. Eur Respir J 1992; 5: 334-342.

20 Delcroix M, Vonk Noordegraaf A, Fadel E, et al. Vascular and right ventricular remodelling in chronic thromboembolic pulmonary hypertension. Eur Respir J 2013; 41: 224-232.

21 Wagenvoort CA. Pathology of pulmonary thromboembolism. Chest 1995; 107: Suppl. 1, 10S-17S.

22 Jamison BM, Michel RP. Different distribution of plexiform lesions in primary and secondary pulmonary hypertension. Hum Pathol 1995; 26: 987-993.

23 Piazza G, Goldhaber SZ. Chronic thromboembolic pulmonary hypertension. N Engl J Med 2011; 364: 351-360.

24 Montani D, Price LC, Dorfmüller P, et al. Pulmonary veno-occlusive disease. Eur Respir J 2009; 33: 189-200.

25 Mitzner W, Wagner EM. Vascular remodeling in the circulations of the lung. J Appl Physiol 2004; 97: 1999-2004.

26 Lovering AT, Stickland MK, Eldridge MW. Intrapulmonary shunt during normoxic and hypoxic exercise in healthy humans. Adv Exp Med Biol 2006; 588: 31-45.

27 Eldridge MW, Dempsey JA, Haverkamp HC, et al. Exercise-induced intrapulmonary arteriovenous shunting in healthy humans. J Appl Physiol 2004; 97: 797-805.

28 Stickland MK, Welsh RC, Haykowsky MJ, et al. Intra-pulmonary shunt and pulmonary gas exchange during exercise in humans. J Physiol 2004; 561: 321-329.

29 Shimizu H, Tanabe N, Terada J, et al. Dilatation of bronchial arteries correlates with extent of central disease in patients with chronic thromboembolic pulmonary hypertension. Circ J 2008; 72: 1136-1141.

30 Orell SR, Hultgren S. Anastomoses between bronchial and pulmonary arteries in pulmonary thromboembolic disease. Acta Pathol Microbiol Scand 1966; 67: 322-338.

31 Fadel E, Wijtenburg E, Michel R, et al. Regression of the systemic vasculature to the lung after removal of pulmonary artery obstruction. Am J Respir Crit Care Med 2006; 173: 345-349.

32 Johnson JE, Perkett EA, Meyrick B. Pulmonary veins and bronchial vessels undergo remodeling in sustained pulmonary hypertension induced by continuous air embolization into sheep. Exp Lung Res 1997; 23: 459-473.

33 Charan NB, Turk GM, Czartolomny J, et al. Systemic arterial blood supply to the trachea and lung in sheep. J Appl Physiol 1987; 62: 2283-2287.

34 Frazier AA, Galvin JR, Franks TJ, et al. From the archives of the AFIP: pulmonary vasculature: hypertension and infarction. Radiographics 2000; 20: 491-524.

35 Charan NB, Carvalho P. Angiogenesis in bronchial circulatory system after unilateral pulmonary artery obstruction. J Appl Physiol 1997; 82: 284-291.

36 Parsons GH, Bommer WJ, Siefkin AD, et al. Drainage routes of bronchial blood flow in anaesthetized sheep. Pulm Pharmacol Ther 2007; 20: 109-111.

37 Charan NB, Thompson WH, Carvalho P. Functional anatomy of bronchial veins. Pulm Pharmacol Ther 2007; 20: $100-103$.

38 Baile EM. The anatomy and physiology of the bronchial circulation. J Aerosol Med 1996; 9: 1-6. 